\title{
Biceps vs extensor carpi radialis recovery in Frankel grades $A$ and $B$ in spinal cord injury patients
}

\author{
L M Kornsgold BA, G J Herbison MD, B F Decena III BS, J F Ditunno Jr MD
}

\section{Department of Rehabilitation Medicine, Jefferson Medical College, Thomas Jefferson University, Philadelphia PA, USA.}

\begin{abstract}
Clinical literature suggests that the wrist extensors show a trend of achieving functional strength earlier than the biceps after spinal cord injury (SCI). Basic research, however, demonstrated that proximal muscles recover earlier than distal muscles after partial denervation. The purpose of this study was to compare biceps to extensor carpi radialis (ECR) recovery of muscle strength in 39 motor complete cervical SCI patients. Biceps $(n=19)$ and ECR $(n=20)$ with a 72 hour or 1 week motor grade of $1 / 5$ were compared. Testing was performed weekly for 1 month, and again at 2, 3, 6 and 12 months post injury. The median recovery times to increase one motor grade were: biceps $=2$ months and ECR $=2.5$ months $(p<0.3)$. The median recovery times to increase two motor grades were: biceps $=2$ months and ECR $=3$ months $(p<0.4)$. In conclusion, there was no significant difference between the rates of recovery of the biceps and the ECR up to 12 months post SCI.
\end{abstract}

Keywords: spinal cord injury; upper limit muscle recovery; wrist extensor muscles; biceps muscle.

\section{Introduction}

Several studies from this center have evaluated the time course of recovery of the muscles of the upper extremities following traumatic spinal cord injury (SCI). ${ }^{1-3}$ These studies have been done to determine the time necessary for denervated muscles to regain functional strength. As stated by Ditunno et al, they are also important for the purpose of determining the effects of various interventions on patient outcome in both acute and chronic settings. ${ }^{1}$ In a prospective study of 32 patients with SCI who had no initial strength (motor grade of $0 / 5)$ in the zone of partial preservation, Wu et $a l^{2}$ were able to support Stauffer's ${ }^{4}$ hypothesis that nerve root function improves at the zone of injury. Comparing motor recovery of patients with an initial motor grade of $1 / 5$ and patients with an

Correspondence: Gerald J Herbison MD, Department of Rehabilitation Medicine, Thomas Jefferson University Hospital, 1015 Walnut Street, Room 617, Philadelphia, PA 19107, USA. initial motor grade of 2/5, Mange et al showed that initial strength is a significant predictor of recovery time. ${ }^{3}$ Ditunno et al combined elements of these two studies to compare motor recovery of patients with some degree of initial muscle strength (motor grade of $1 / 5$ or $2 / 5$ ) to patients with no initial muscle strength (motor grade of $0 / 5) .{ }^{1}$ In this multicenter study, it was concluded that muscles with some initial strength were able to regain a functional level earlier than muscles with no initial strength.

All three of the above mentioned studies were designed to evaluate motor recovery solely as a function of initial muscle strength. Ditunno and associates demonstrated that the motor recovery of the extensor carpi radialis (ECR) recovered to at least $3 / 5$ if the initial motor grade of the ipsilateral biceps on the initial post-SCI manual muscle test was $3 / 5 .^{5}$ While Ditunno and his associates did not specifically compare motor recovery between two different muscles, they suggested that the wrist extensors with some initial motor power showed a 
trend of achieving functional strength earlier than the biceps with some initial motor power. ${ }^{1}$ If true, this finding would not be consistent with human ${ }^{6}$ and animal $^{7}$ studies which suggest a direct correlation between nerve length and recovery time after partial denervation. The present study was designed to test the hypothesis that the ECR begins to recover muscle strength earlier in the post-SCI period than the biceps after partial denervation.

\section{Methods}

This prospective study followed motor complete (Frankel A and B) subjects $^{8}$ with cervical spinal cord injury (SCI) and gun shot wounds (GSW) to 6 months post injury. Hereafter, both SCI and GSW subjects will be grouped together as SCI subjects because there was no difference in the rates of recovery. Comparisons were made for the times at which the biceps and extensor carpi radialis (ECR) increased by one and two manual muscle testing (MMT) grades. Recovery of muscle strength was determined by manual muscle testing (MMT) utilizing a grading scale of the Medical Research Council (MRC) ${ }^{9}$ and the American Spinal Injury Association $(\mathrm{ASIA})^{10}(0,1,2,3,4$, and 5) and incorporating half grades $(0.5)$ to further identify strength between the basic grades. Therefore, in this study, the muscles tested were graded $0,1,1.5,2,2.5,3,3.5,4,4.5$, and 5 where: $0=$ total paralysis; $1=$ some palpable or visible contraction of muscle but no movement of body part; $1.5=$ movement but not through a full range of motion (ROM) with gravity eliminated; $2=$ movement through a full ROM with gravity eliminated; $2.5=$ same as 2 but also withstanding minimal resistance; $3=$ movement through a full ROM and against gravity; $3.5=$ same as 3 and withstanding minimal resistance; $4=$ same as 3 and withstanding moderate resistance; $4.5=$ same as 3 and withstanding almost normal resistance; $5=$ same as 3 and withstanding maximum (normal) resistance. This grading system has been utilized in previous studies reported from our center. ${ }^{3,11-13}$ At our center, interrater reliability of the MMT was determined by having four raters from our trained staff test six subjects with SCI on five upper extremity muscles. Data were analyzed by a generalized Kappa. ${ }^{14}$ There was substantial agreement between the raters for the upper extremity muscles $(k=0.64)$. Manual muscle testing, as a measure of strength, for grading key muscles has also been found to be reliable at other centers. ${ }^{15,16}$ Approximately 520 patients were admitted to the Regional Spinal Cord Injury Center of Delaware Valley (RSCICDV) between March 1988 and January 1992. Twenty-five percent of the patients in our center had complete cervical spinal cord injuries as defined by ASIA $^{10}$ and Frankel. ${ }^{8}$ To be included in the study, every subject must have been admitted to the RSCICDV within 1 week of injury, and must also have undergone a neurological examination and MMT between 3 and 7 days post injury. At the time of the 72 hour MMT or 1 week MMT, each subject must have presented as a motor complete (Frankel classes $\mathrm{A}$ and B $)^{8}$ cervical spinal cord injury with the most rostral muscle in the zone of partial preservation (ZPP) being either the biceps (C4 injury) or the extensor carpi radialis (C5 injury). ${ }^{10}$

The muscle to be studied must have had a muscle grade of $1 / 5$. We followed the MRC system of muscle testing where no attempt was made to separate the extensor carpi radialis longus (ECRL) from the extensor carpi radialis brevis (ECRB); the two muscles were tested as one muscle. If a subject's biceps were to be studied, then that subject must have had normal pin sensation to the level of $\mathrm{C} 4$ on the same side. This criteria eliminated patients who had sustained higher levels of injury with extended ZPPs. If a subject's ECR was to be studied, then that subject must also have had a motor grade greater than or equal to $3 / 5$ in the ipsilateral biceps. It is important to note that the neurological levels assigned to patients at the time of admission were not used as criteria for inclusion into or exclusion from the study. This choice was made because some SCI patients had changes in neurological level between the time of admission and 72 hours post injury. Brown and associates have shown that the 72 hour 
MMT is a better predictor of recovery in the ZPP than the MMT done on the day of injury. ${ }^{13}$ Herbison et al ${ }^{17}$ showed that the 72 hour and 1 week periods are similar and represent the best times to evaluate strength for research and prognostic purposes. Patients were excluded from the study if a fracture of the extremity to be studied was present. Other physical or psychological impairments that would affect evaluation also disqualified patients from participating in the study, as would an inability to follow instructions. Informed consent was obtained from all of the patients to conduct MMT testing procedures and to utilize the information from the testing in a publication.

For the purpose of this study, the subjects, on admission, were classified ${ }^{10}$ as neurological levels C4 $(n=30)$ and C5 $(n=9)$ with the following motor levels C4 $(n=17)$ and C5 $(n=22)$. When reevaluated between 3 and 7 days post injury, the patients were reclassified based on the above described rigid criteria for inclusion into the study. The 39 subjects were regrouped as neurological levels C4 $(n=34)$ and C5 $(n=5)$ with the following motor levels C4 $(n=20)$ and C5 $(n=19)$. The group of which 38 were male and one was female, ranged in age from 16 to 68 years old with an average age of 29 years old. The causes of injuries were $7 / 39$ gun shot wound, 14/39 diving, 9/39 motor vehicle accident, $4 / 39$ fall, one train accident, one dirt bike, one person-to-person contact, one ice hockey, and one waterslide.

Two groups were identified based on the results of MMT done between 3 and 7 days post injury. Group B $(n=19)$ comprised those subjects with an initial biceps motor grade of $1 / 5$ or $1+/ 5$. Group $\mathrm{E}(n=20)$ consisted of those subjects with an initial ECR motor grade of $1 / 5$ or $1+/ 5$. Immediately post SCI, six of the subjects in group B were on high dose steroids, as well as 14 of the subjects in group E. ${ }^{18}$

A number of subjects from both groups underwent surgery at some time since their accidents. Twelve of the 19 subjects in group $B$ had operations: four underwent anterior cervical fusion (ACF); four underwent posterior cervical fusion (PCF); two underwent $\mathrm{ACF}$ and PCF; one underwent $\mathrm{ACF}$ and vertebral fracture repair (VFR); and one underwent PCF and VFR. Fifteen of the 20 subjects in group $\mathrm{E}$ had operations: four underwent PCF; two underwent $\mathrm{ACF}$ and PCF; four underwent ACF, PCF, and excision vertebral disc (EVD); one underwent $\mathrm{ACF}, \mathrm{EVD}$, and partial osteoectomy (PO); one underwent ACF, PCF, and PO; one underwent $\mathrm{ACF}$ and $\mathrm{PO}$; one underwent $\mathrm{ACF}, \mathrm{PCF}, \mathrm{EVD}$, and PO; and one underwent $\mathrm{PO}$ and posterior lumbosacral fusion (PLSF).

Subjects in both groups were retested weekly for 1 month and again at 2, 3,6 and 12 months post SCI. Two of the subjects from group $\mathrm{B}$ and four of the subjects from group $\mathrm{E}$ were followed to 12 months. Recovery of muscle strength as indicated by an increase in motor grade was followed to two endpoints. For each group, recovery was followed to an increase of one motor grade and an increase of two or more motor grades with no more than one subsequent decrease in motor grade nor any single decrease in motor grade greater than $1 / 5$ at any subsequent time. For example, if a subject received a motor grade of $3 / 5$ at 2 months post injury, he/she was said to have achieved success at 2 months if he/she had no more than one subsequent motor grade less than $2 / 5$. Recovery time was defined as the time interval at which a given subject achieved success. The statistical analysis was performed utilizing the median test and the Kruskal-Wallis test. ${ }^{19}$ The statistical analysis was done using the data up to 12 months. There were too few patients receiving either initial high dose steroids and/or no surgery to separately analyze their effects on recovery. Differences in the time to recovery comparing the biceps to the extensor carpi radialis were considered significant if $p \leqslant 0.05$.

\section{Results}

\section{Recovery of one motor grade}

The recovery times for the subjects in group $\mathrm{B}(n=19)$ ranged from 1 week to 12 months post injury (Table I, Fig 1). The median recovery time for this group was calculated to 2 months. Recovery times ranged from 1 week to 6 months for the 20 
Table I Recovery time (biceps)

\begin{tabular}{rccccc}
\hline Patient & $\begin{array}{c}\text { Time to recover } \\
1 \text { motor grade }\end{array}$ & $\begin{array}{c}\text { Time to recover } \\
2 \text { motor grades }\end{array}$ & $\begin{array}{c}\text { MMT } \\
1 \text { month }\end{array}$ & $\begin{array}{c}\text { MMT } \\
3 \text { months }\end{array}$ & $\begin{array}{c}\text { MMT } \\
\text { months }\end{array}$ \\
\hline 1 & 2 months & 2 months & $1+$ & $3+$ & $3+$ \\
2 & 2 weeks & 1 month & 3 & $3+$ & $2+$ \\
3 & 3 months & 3 months & $1+$ & 4 & $4+$ \\
4 & 6 months & 6 months & $1+$ & $1+$ & 4 \\
5 & 2 months & 2 months & $1+$ & 3 & $3+$ \\
6 & 12 months & 12 months & 1 & 0 & $1+$ \\
7 & 2 months & 2 months & $1+$ & $4+$ & 5 \\
8 & 3 weeks & 3 weeks & 4 & $4+$ & $4+$ \\
9 & 2 weeks & 2 weeks & NT & $2+$ & 4 \\
10 & 2 months & 2 months & $1+$ & $3+$ & $3+$ \\
11 & 2 months & 2 months & NT & 4 & $2+$ \\
12 & 3 weeks & 6 months & $2+$ & NT & $4+$ \\
13 & 2 months & 2 months & NT & 4 & $4+$ \\
14 & 3 months & 3 months & 0 & $3+$ & 4 \\
15 & 2 months & 6 months & $1+$ & $1+$ & 4 \\
16 & 1 month & 12 months & 2 & 2 & $1+$ \\
17 & 2 months & 2 months & 1 & NT & $4+$ \\
18 & 1 week & 2 weeks & 4 & NT & NT \\
19 & 1 week & 2 weeks & $4+$ & 5 & 5 \\
\hline
\end{tabular}

$\mathrm{MMT}=$ manual muscle test $; \mathrm{NT}=$ not testable.

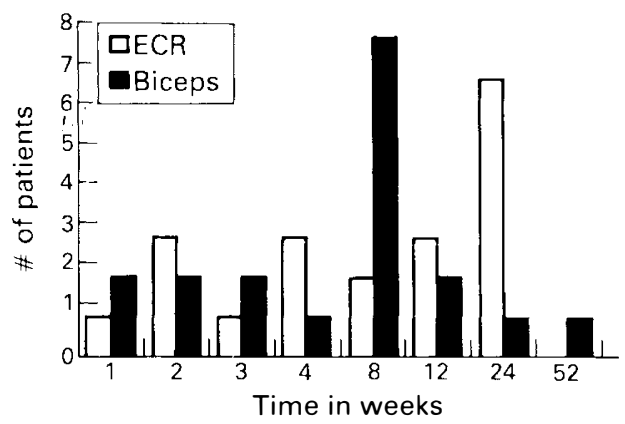

Figure 1 ECR and biceps: time to recover one motor grade for patients at each time period (not cumulative).

subjects in group E (Table 2, Fig 2). The median recovery time calculated for this group was $2 \frac{1}{2}$ months. The significance in recovery times to 12 months between the biceps and the ECR was $p<0.3$.

\section{Recovery of two motor grades}

Seventeen of the 19 subjects in group B achieved success by 6 months with the earliest recovery time being 2 weeks. The other two subjects did not achieve success until 12 months (Table 1, Fig 1). The median recovery time for these subjects was 2 months. For group $\mathrm{E}(n=20)$, four subjects did not achieve success by 6 months post injury but they achieved success by 12 months. The recovery times for this group ranged from 2 weeks to 12 months with the median calculated as 3 months (Table 2, Fig 2). There was no significant difference in median recovery times between the biceps and the ECR $(p<0.4)$.

\section{Discussion}

Based on the results of this prospective study, we reject the hypothesis that the ECR begins to recover muscle strength earlier in the post-SCI period than the biceps after partial denervation. There was no significant difference in recovery between the biceps and ECR up to 12 months post SCI. Ditunno and associates have described possible mechanisms of motor recovery occurring in three different windows of time post SCI. ${ }^{5}$ The different windows of recovery were not distinct. The mechanisms that explained the recovery of 
Table II Recovery time (extensor carpi radialis)

\begin{tabular}{rccccc}
\hline Patient & $\begin{array}{c}\text { Time to recover } \\
1 \text { motor grade }\end{array}$ & $\begin{array}{c}\text { Time to recover } \\
2 \text { motor grades }\end{array}$ & $\begin{array}{c}\text { MMT } \\
1 \text { month }\end{array}$ & $\begin{array}{c}\text { MMT } \\
\text { months }\end{array}$ & $\begin{array}{c}\text { MMT } \\
\text { months }\end{array}$ \\
\hline 1 & 6 months & 6 months & 1 & 1 & $3+$ \\
2 & 6 months & 6 months & 0 & 1 & $3+$ \\
3 & 6 months & 12 months & $1+$ & $1+$ & $2+$ \\
4 & 1 week & 2 weeks & 3 & $4+$ & 4 \\
5 & 2 weeks & 3 months & $1+$ & 3 & 3 \\
6 & 1 month & 1 month & 3 & $3+$ & 4 \\
7 & 3 weeks & 3 weeks & $3+$ & NT & 4 \\
8 & 3 months & 6 months & 1 & $2+$ & 3 \\
9 & 6 months & 6 months & 0 & $1+$ & $3+$ \\
10 & 1 month & 1 month & 3 & $3+$ & 5 \\
11 & 3 months & 3 months & $1+$ & $3+$ & NT \\
12 & 6 months & 12 months & 1 & 1 & $2+$ \\
13 & 3 months & 3 months & $1+$ & $3+$ & $3+$ \\
14 & 2 months & 2 months & 1 & NT & 3 \\
15 & 6 months & 12 months & 0 & $1+$ & $2+$ \\
16 & 2 weeks & 2 weeks & 2 & $3+$ & $3+$ \\
17 & 2 months & 2 months & NT & $3+$ & $3+$ \\
18 & 6 months & 12 months & 1 & NT & 2 \\
19 & 1 month & 1 month & $3+$ & NT & $3+$ \\
20 & 2 weeks & 2 months & $2+$ & $4+$ & NT \\
\hline
\end{tabular}

$\mathrm{MMT}=$ manual muscle test $; \mathrm{NT}=$ not testable.

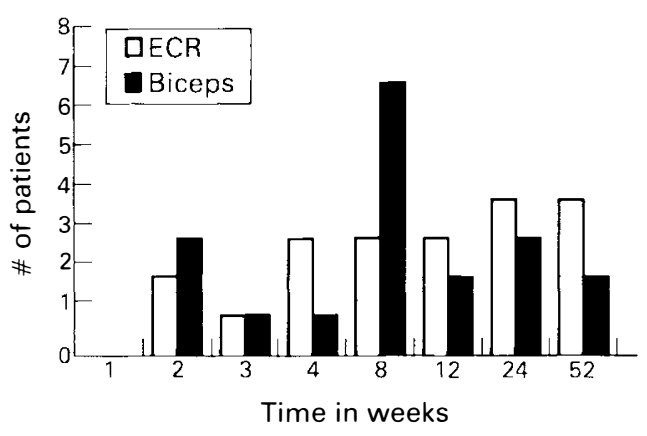

Figure 2 ECR and biceps: time to recover two motor grades for patients at each time period (not cumulative).

strength overlapped the different time windows which Ditunno and associates characterized as early, middle, and late recovery phases. ${ }^{5}$ Mechanisms for improvement during these phases included reversal of peripheral ${ }^{20-27}$ and central nervous system (CNS) conduction block, ${ }^{28}$ CNS plasticity, ${ }^{29-32}$ peripheral ${ }^{33-39}$ and CNS sprouting, ${ }^{40,41}$ muscle fiber hypertrophy, ${ }^{42}$ and axonal regeneration. ${ }^{43-46 .}$

A reversal of peripheral conduction block in the first few weeks post SCI could have occurred at-the root level just outside the spinal cord. Microvasculature compression could have caused ischemia as a pathophysiological mechanism of conduction block. $^{20,21}$ It has been suggested that the conduction failure from ischemia has a metabolic basis,,$^{22,23}$ and that its reversal results from restoration of blood flow. Reversal of ischemia and correction of normal neuronal metabolism may have been the explanation for the early recovery phase in two patients from group B (patients 18 and 19) and one patient from group $E$ (patient 4) in this study who recovered one motor grade within 7 days post SCI (Tables I, II).

Fowler et al suggested that conduction block could be caused by disruption of the nodes of Ranvier due to the pressure gradient created in a compression injury. ${ }^{24}$ There is a pressure gradient in the nerve between its compressed and uncompressed parts. The compression caused a longitudinal movement of the axon and its myelin from the compression site as well as a disruption of the nodes due to the dislocation of the 
paranodal myelin with the lesions being concentrated at the edges of the compression zone. ${ }^{25}$ Ochao et al also demonstrated that compression caused displacement of the node of Ranvier with paranodal myelin and invagination paranodal demyelination followed by degeneration of myelin. ${ }^{25}$ Regardless of whether ischemia or myelin disruption or both accounted for the conduction block, the compression was responsible for the loss of conduction. ${ }^{26} \mathrm{Re}$ covery from conduction block may occur within hours, days, several weeks or a few months and it does not follow a proximal to distal course or any specific pattern of recovery. ${ }^{27}$ Since reversal of conduction block does not follow a specific pattern of recovery, this mechanism could explain the similar rates of recovery of the biceps and the ECR found in the present study.

Not only can reversal of conduction block be found in the peripheral nervous system, but this mechanism of recovery can also be seen at the level of the CNS. Recent studies have shown that remyelination should lead to clinical improvement on the basis of reversal of conduction block in the central nervous system. ${ }^{28}$ Therefore, recovery within the spinal cord of the upper motor neurons and/or recovery of conduction of the intraspinal cord lower motor neurons could contribute to the recovery of strength post SCI.

Another mechanism in SCI recovery is the plasticity of the central nervous system. Plasticity can occur by reinstitution of conduction in demyelinated axons in the absence of remyelination and/or new transmission at previously inactive synaptic sites. ${ }^{29,30}$ The structure of the axolemma is not static but highly dynamic, and plasticity can endow the axon with its capability to reorganize and restore conduction in demyelinated axons so that action potentials can transverse the zone without myelin. ${ }^{29,30}$ At a cellular level, conduction through demyelinated axon regions can be facilitated by several mechanisms, including development of excitability in demyelinated regions, and impedance matching. ${ }^{30}$. The $\mathrm{Na}^{+} \mathrm{K}^{+}$channels that were inactive become active. Secondly, Dimitrijevic suggested that spinal cord plasticity as the result of the modifica- tion of synaptic transmission after injury might play a part in the recovery process. ${ }^{31}$ After a spinal cord injury, there may be a transfer of function from undamaged pathways responsible for discrete muscle activity to neurons that lost suprasegmental control due to the SCI. This transfer of control may result in recovery of function of upper motor neuron denervated lower motor neurons. ${ }^{32}$ The time period in which plasticity may play a part is not known. In the present study, if the mechanism of plasticity contributed to recovery, then it operated on a similar basis for proximally and distally innervated muscles.

There are two other mechanisms of recovery that may account for improvement in strength post SCI: sprouting in the peripheral and central nervous systems and muscle fiber hypertrophy of intact motor units. Partial denervation is followed by sprouting of intact axons which could lead to peripheral reinnervation of the adjacent denervated muscle fibers. ${ }^{33-36}$ The stimulus for sprouting reportedly is a transient signal which can be effective soon after denervation, but might decrease subsequently. ${ }^{37}$ Recently, Massey \& Sanders ${ }^{34}$ described the time course and dynamics of denervation and reinnervation following nerve trauma. According to their study, fibrillations were seen as early as 15 days post injury, peaked at 29 days, and had decreased again by 37 days post injury. In the same study, fiber density, evidence of peripheral sprouting, increased at 21 days and jitter increased at 15 days post injury, and both reached maximal values at 37 days. These results were interpreted to indicate that the functional contact had been made between collateral sprouts and muscle by 21 days. This type of recovery occurred later than reversal of conduction block and could explain the recovery of strength in the biceps and the ECR as early as 1 month post SCI. Patients who did not have complete recovery probably had such a marked loss of motor units that the remaining intact motor units could not compensate for the loss of motor neuron pool. ${ }^{38}$ Hansen \& Ballantyne reported that reinnervation in motor neuron disease was sufficient to compensate completely for the loss of up to $50 \%$ of the 
motor neuron pool supplying the muscle. ${ }^{38}$ Gordon and her coworkers found that single motor units enlarged up to five times their original size in the hand muscles of quadriplegic subjects, resulting in the ability to compensate for up to $80 \%$ of motor neuron loss. ${ }^{36}$ Most of the subjects in the present study did not recover completely. Therefore, these subjects presumably had greater than $50-80 \%$ denervation at the time of SCI.

In a study of peripheral sprouting utilizing botulinum toxin, Pestronk \& Drachman reported a 20 - to 30 -fold higher incidence of sprouting in the rhomboid than in the soleus and lumbrical muscles of rats, within the same time period after the administration of the botulinum. ${ }^{7}$ They suggested that the variations were related to differences in the intrinsic capacities of axons, and that sprouting and repair mechanisms may be less efficient in longer nerves resulting in a reduced ability to recover. Electrophysiologic studies in humans tend to support the proximal to distal relationship of peripheral sprouting as determined by the disappearance of fibrillation potentials, first in proximal and then in distal muscles after partial denervation. ${ }^{6}$ In the present study, there was no significant difference in the recovery of biceps vs ECR. This may be due to the comparable length of the axons supplying both muscles. A study of muscles innervated by axons of markedly different lengths may support Pestronk \& Drachman's finding in animals of early recovery of proximal vs distal muscles. ${ }^{7}$

There is also the possibility of axonal sprouting in the CNS axons. Sprouting is the response of the uninjured axons to the partially denervated area of the central nervous system. ${ }^{31}$ Kalil \& Reh suggested that recovery of function in the mammalian CNS involved sprouting of intact axons into a foreign territory that had been denervated by damage to its normal inputs. ${ }^{39}$ More recent studies have shown that sprouting occurred locally into denervated regions of the spinal cord and that sprouting appeared to arise in response to local signals within the cord and not to long distance cues. ${ }^{40}$

Muscle fiber hypertrophy is another mechanism of recovery that may occur concomitantly with peripheral sprouting. ${ }^{5}$ Muscle fiber hypertrophy has been described in patients who had poliomyelitis after an estimated $41-87 \%$ loss of lower motor neurons. ${ }^{41}$ Although Ditunno et al ${ }^{5}$ suggested that muscle fiber hypertrophy contributed to the recovery of strength between 2 and 6 months post SCI, it would be of interest to identify the exact time course and degree of muscle fiber hypertrophy after SCI.

Axonal regeneration, a mechanism responsible for late recovery, can be modified by local conditions. ${ }^{27}$ Axonal regeneration could occur if there was damage to the root, but not if there was damage to the anterior horn cell. Regeneration of an axon begins with growth of neuronal branches origination from intact Schwann cell columns. ${ }^{26}$ In their study, Bowden \& Sholl showed that the rate of regeneration declined with distance. ${ }^{42}$ Several studies of motor fiber regeneration have proposed different numerical values for the actual mean rate. Rates calculated by clinical observation of the return of voluntary motor activity range from $1.7 \mathrm{~mm} / \mathrm{day}^{29,43}$ to $4.4 \mathrm{~mm} / \mathrm{day},{ }^{29,44}$ while electromyographic studies have shown regeneration rates that range in value from $1 \mathrm{~mm} /$ day $^{29,45}$ to $3.4 \mathrm{~mm} /$ day. ${ }^{29,46}$ Clinicians, in general, use the rate of $1 \mathrm{~mm} /$ day (approximately $1 \mathrm{in} /$ month) in practice. ${ }^{27}$ Considering that the distance between the spinal cord and both the biceps and the ECR is at least 12 inches in an average-sized adult, this mechanism could explain potential recovery after 12 months post SCI. This mechanism may explain recovery, but not in the time frame of the patients followed in the present study.

In conclusion, there was no significant difference in recovery between biceps and ECR although there was a trend of earlier recovery of the biceps compared to the ECR. Since there was a similar pattern of recovery in the biceps and ECR, it appears justifiable to group biceps and ECR with a 1/5 MMT grade for the purposes of evaluating recovery post SCI. Based on Pestronk \& Drachman's ${ }^{7}$ study, it would be of interest to further evaluate recovery of strength of biceps vs more distal muscles to determine if proximal muscles recover at a faster rate 
than distal muscles due to peripheral sprouting.

\section{Acknowledgements}

We wish to express our gratitude to Gregory $\mathrm{L}$ Purnsley for the statistical analysis, Barbara E
Wolff for the data collection, and Patt Williams for the preparation of the manuscript. This study was supported in part by awards from the National Institute on Disability and Rehabilitation Research to the Regional Spinal Cord Injury Center of Delaware Valley (\#G008535135) and the National Rehabilitation Research and Training Center in Spinal Cord Injury (\#H133B80017).

\section{References}

1 Ditunno JF Jr, Stover SL, Freed MM, Ahn JH (1992) Motor recovery of the upper extremities in traumatic quadriplegia: A multi-center study. Arch Phys Med Rehabil 73: 431-436.

2 Wu L, Marino RJ, Herbison GJ, Ditunno JF Jr (1992) Recovery of zero-grade muscles in the zone of partial preservation in motor complete quadriplegia. Arch Phys Med Rehabil 73: 40-43.

3 Mange KC, Marino RJ, Gregory PC, Herbison GJ, Ditunno JF (1992) Course of motor recovery in the zone of partial preservation in spinal cord injury. Arch Phys Med Rehabil 73: 437-441.

4 Stauffer ES (1984) Neurologic recovery following injuries to the cervical spinal cord and nerve roots. Spine 9: $532-534$.

5 Ditunno JF Jr, Sipski ML, Posuniak EA, Chen YT, Staas WE Jr, Herbison GJ (1987) Wrist extensor recovery in traumatic quadriplegia. Arch Phys Med Rehabil 68: 287-290.

6 Wilbourn AJ, Aminoff MJ (1988) AAEE Minimonograph \#32: The electrophysiologic examination in patients with radiculopathies. Muscle Nerve 11: 1099-1114.

7 Pestronk A, Drachman DB (1988) Motor nerve outgrowth: Reduced capacity for sprouting in the terminals of longer axons. Brain Res 463: 218-222.

8 Frankel H, Hancock D, Hyslop G, Melzak J, Michaelis L, Unger G et al (1969) The value of postural reduction in the initial management of closed injuries to the spine with paraplegic and tetraplegia. Paraplegia 7: 179-192.

9 Medical Research Council (1943) Aids to the investigation of peripheral nerve injuries. War Memorandum, 2nd ed. London.

10 American Spinal Injury Association (1992) Standards for Neurologic and Functional Classification of Spinal Cord Injury Patients. Chicago.

11 Browne BJ, Jacobs SR, Herbison GJ, Ditunno JF (1993) Pin sensation as a predictor of extensor carpi radialis motor recovery at the zone of partial preservation in spinal cord injured subjects. Arch Phys Med Rehabil 74: 14-18.

12 Zafonte RD, Demangone DA, Herbison GJ, Ditunno JF (1991). Daily self care in quadriplegic subjects. J Neurol Rehabil 1: 17-24.

13 Brown PJ, Marino RJ, Herbison GJ, Ditunno JF (1991) The 72-hour exam on a predictor of recovery in motor complete quadriplegia. Arch Phys Med Rehabil 72: 546-548.

14 Fleiss JL (1981) Statistical Methods for Rates and Proportions. 2nd ed. John Wiley and Sons, New York.

15 Aitkens S, Lord J, Bernauer E, Fowler WM, Lieberman JS, Berck P (1989) Relationship of manual muscle testing to objective strength measurements. Muscle Nerve 12: 173-177.

16 Florence JM, Pandya S, King WM, Robinson JD, Baty J, Miller JP et al (1992) Intrarater reliability of manual muscle test (Medical Research Council Scale) grades in Duchene's muscular dystrophy. Phy Ther 72: $115-126$

17 Herbison GJ, Zerby SA, Cohen ME, Marino RJ, Ditunno JF (1992) Motor power difference within the first two weeks post-S.CI in cervical spinal cord injured quadriplegic subjects. J Neurotrauma 9: 373-380.

18 Bracken MB, Shepard MJ, Collins WF et al (1990) A randomized, controlled trial of methylprednisolone or naloxone in the treatment of acute spinal cord injury. $N$ Engl J Med 322: 1405-1411.

19 Zar JH (1984) Biostatistical Analysis. Prentice-Hall, Englewood Cliffs.

20 Sunderland S (1991) Nerve Injuries and Their Repair: A Critical Appraisal. Churchill Livingstone, Edinburgh.

21 Denny-Brown MB, Brenner C (1944) Paralysis of nerve induced by direct pressure and by tourniquet. Arch Neurol Psychiatry 51: 1-26.

22 Parry GJ, Cornblath DR, Brown MJ (1985) Transient conduction block following acute peripheral nerve ischemia. Muscle Nerve 8: 409-412.

23 Ropert A, Metral S (1990) Conduction block in neuropathies with necrotizing vasculitis. Muscle Nerve 13: 102-105.

24 Fowler TJ, Danta G, Gilliatt RW (1972) Recovery of nerve conduction after a pneumatic tourniquet: Observations on the hind-limb of the baboon. J Neurol Neurosurg Psychiatry 35: 638-647.

25 Ochoa J, Fowler TJ, Gilliatt RW (1972) Anatomical changes in peripheral nerves compressed by a pneumatic tourniquet. $J$ Anat 113: 433-455. 
26 Lundborg G (1988) Nerve Injury and Repair. Churchill Livingstone, Edinburgh.

27 Seddon H (1975) Surgical Disorders of the Peripheral Nerves. Churchill Livingstone, Edinburgh.

28 Young W (1989) Recovery mechanisms in spinal cord injury: Implications for regenerative therapy. In: Seil FJ, editor. Neural Regeneration of Transplantation. Frontiers of Clinical Neuroscience 6. AR Liss, New York: $157-169$.

29 Waxman SG (1988) Biophysical mechanisms of impulse conduction in demyelinated axons. In: Waxman SG, editor. Advances in Neurology, Volume 47. Functional Recovery in Neurological Disease. Raven Press, New York: $185-213$.

30 Waxman SG (1992) Demyelination in spinal cord injury and multiple sclerosis: What can we do to enhance functional recovery? J Neurotrauma 9: S105-S117.

31 Dimitrijevic MR (1988) Residual motor functions in SCI. In Waxman SG, editor. Advances in Neurology, Volume 47. Functional Recovery in Neurological Disease. Raven Press, New York: 139-155.

32 Goldenberger ME (1988) Spinal cord plasticity, regeneration and recovery of function. Curr Opin Neurol Neurosurg 1: 592-600.

33 Herbison GJ, Jaweed MM, King LJ, Ditunno JF Jr (1983) Evaluation of peripheral reinnervation of muscle in spinal nerve. Arch Phys Med Rehabil 64: 497.

34 Massey JM, Sanders DB (1991) Single-fiber EMG demonstrates reinnervation dynamics after nerve injury. Neurology 41: 1150-1151.

35 Marino RJ, Herbison GJ, Ditunno JF (1992) Peripheral sprouting in zone of partial preservation in motor complete spinal cord injury. J Am Paraplegia Soc 15: 122.

36 Gordon T, Yang JF, Ayer K, Stein RB, Tyreman N (1993) Recovery potential of muscle after partial denervation: A comparison between rats and humans. Brain Res Bull 30: 477-482.

37 Thompson W, Jansen JKS (1977) The extent of sprouting of remaining motor units in partly denervated immature and adult rat soleus muscle. Neuroscience 2: 523-535.

38 Hansen S, Ballantyne JP (1978) A quantitative electrophysiological study of motor neurone disease. J Neurol Neurosurg Psychiatry 41: 773-783.

39 Kalil K, Reh T (1979) Regrowth of severed axons in the neonatal central nervous system: Establishment of normal connections. Science 205: 1158-1160.

40 Kuang RZ, Kalil K (1990) Specificity of corticospinal axon arbors sprouting into denervated contralateral spinal cord. J Compar Neurol 302: 461-472.

41 Einarsson G, Grimsby G, Stalberg E (1990) Electromyographic and morphological functional compensation in late poliomyelitis. Muscle Nerve 13: 165-171.

42 Bowden REM, Sholl DA (1950) The advance of functional recovery after radial nerve lesions in man. Brain 73: 251-266.

43 Seddon HJ, Medawar PB, Smith H (1943) Rate of regeneration of peripheral nerves in man. J Physiol 102: 191-215.

44 Sunderland S (1978) Nerves and Nerve Injuries. Churchill Livingstone, Edinburgh.

45 Isch F, Isch-Treussard C, Jesel M (1968) EMG findings on reinnervation in peripheral nerve lesions. Electroencephalogr Clin Neurophysiol 25: 404-405.

46 Jasper HH (1946) The rate of reinnervation of muscle following nerve injuries in man as determined by the electromyogram. Trans $R$ Soc Med Can 40: 81-92. 